\title{
Perception of animacy from the motion of a single object
}

\author{
Patrice D Tremoulet, Jacob Feldman \\ Department of Psychology and Center for Cognitive Science, Rutgers University, New Brunswick, \\ NJ 08903, USA; e-mail: pollyt@ruccs.rutgers.edu \\ Received 2 December 1999, in revised form 16 May 2000
}

\begin{abstract}
We demonstrate that a single moving object can create the subjective impression that it is alive, based solely on its pattern of movement. Our displays differ from conventional biological motion displays (which normally involve multiple moving points, usually integrated to suggest a human form) in that they contain only a single rigid object moving across a uniform field. We focus on motion paths in which the speed and direction of the target object change simultaneously. Naive subjects' ratings of animacy were significantly influenced by (i) the magnitude of the speed change, (ii) the angular magnitude of the direction change, (iii) the shape of the object, and (iv) the alignment between the principal axis of the object and its direction of motion. These findings are consistent with the hypothesis that observers classify as animate only those objects whose motion trajectories are otherwise unlikely to occur in the observed setting.
\end{abstract}

\section{Introduction}

It is well known that the human visual system can integrate the motions of a number of point-lights into a percept of a human form walking, dancing (Johannson 1973), or performing a wide variety of other actions (Dittrich 1993). This ability extends to point-light displays of non-human animals locomoting (Mather and West 1993) but not to articulated motions of inanimate objects, at least under impoverished conditions (Shiffrar et al 1997). Perception of biological motion has usually been associated with integration of articulated joint motions (Hoffman and Flinchbaugh 1982; Webb and Aggarwal 1982), and is known to respect biomechanical motion constraints (Chatterjee et al 1996). Some authors have hypothesized that the visual system automatically detects events in which the observable kinetic energy increases (Bingham et al 1995), or is otherwise not conserved (Stewart 1982), which might imply the presence of an animate entity with hidden energy sources. This suggests that the motion of a single point might create an impression of animacy, in particular when the motion path in some way suggests volitional or intentional control.

Indeed, classic demonstrations by Heider and Simmel (1944) and Michotte (1963) have illustrated that observers sometimes attribute elaborate motivations, intentions, and goals to individual rigid objects, based solely on the pattern of their movements. The visual system of the frog is thought to reflexively classify visual targets into animate (potential food) and inanimate categories (Lettvin et al 1959), again based solely on motion. Developmental research suggests that pre-verbal human infants make a fundamental ontological distinction between animate and inanimate agents, with motion cues providing a decisive influence on their classification of objects into these categories (Dasser et al 1989; Gelman 1990; Premack 1990; Mandler 1992; Spelke et al 1995). Recently, researchers in computational vision (Mann et al 1997) have modeled the interpretation of dynamic displays using a default logic in which animacy is only attributed to those objects whose motion cannot be explained otherwise.

In a small number of studies adults' judgments of the animacy of rigid moving targets have been directly investigated (Bassili 1976; Stewart 1982; Dittrich and Lea 1994; Bingham et al 1995; Gelman et al 1995). However, these studies included long, complex motion trajectories (Bingham et al 1995), interactions among multiple elements 
(Bassili 1976; Dittrich and Lea 1994), or complex static visual environments (Stewart 1982; Gelman et al 1995), making it difficult to isolate the motion factors essential to the judgment of animacy. In our study we focus on the motion of a single particle moving on a featureless dark background, and attempt to investigate systematically the motion parameters that contribute to the subjective impression of animacy.

We reasoned, following Gelman et al (1995), that observers who are asked to decide if a moving object is animate would base their decision upon inferences about the causes of its motion. For example, if a motion appears to be self-generated, ie caused by an internal rather than an external energy source, then it should produce an impression of animacy. We decided to concentrate upon simple trajectories that include a change in velocity, since constant-velocity motion requires no energy transfer. ${ }^{(1)}$ Of course, inanimate objects can, and frequently do, change velocity-balls roll off tabletops, bounce off floors, etc. However, most inanimate velocity changes involve direct contact with other visible entities (tables, floors), although some do not, eg leaves blown by the wind. Nonetheless, a change in velocity implies a transfer of energy, and if this velocity change is witnessed in a featureless environment, then the source of this velocity change can not be a visible external entity. Observers must thus decide if an invisible external energy source or a hidden internal source of energy caused the change in velocity.

We hypothesized that simultaneous changes in both speed and motion directionoccurring in a uniform, featureless environment - would lead to animate interpretations. In the absence of any supporting context, these trajectories cannot normally be accounted for by inanimate motion sources common in the environment, and hence might trigger the inference of an animate agent. This led us to manipulate direction change (the angular change in motion direction), and speed change (the ratio of final speed to initial speed). In addition, we compared three shape/alignment conditions (figure 1). In the dot condition, the target was a circular patch. In the aligned condition, the target was a narrow rectangle always oriented along the direction of motion. In the misaligned condition, the target was a rectangle always oriented along the

(a)

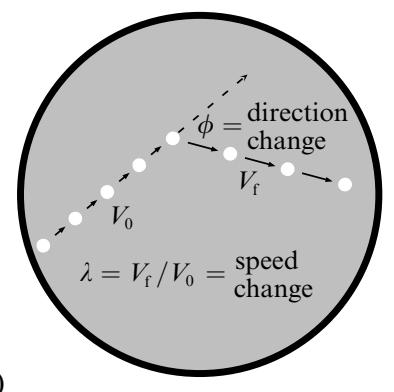

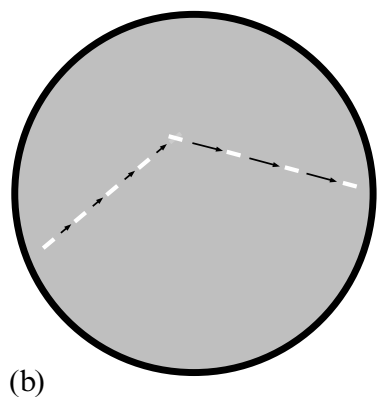

(b)

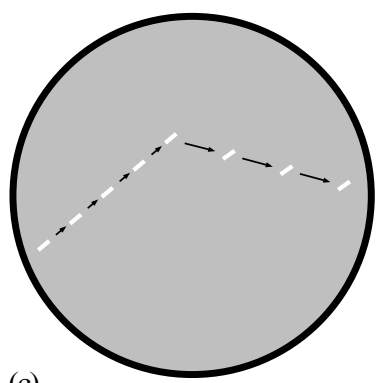

(c)

Figure 1. Shape/alignment conditions: (a) dot condition (also shown are the motion parameters); (b) aligned condition; (c) misaligned condition. In all conditions, a particle initially moves in a randomized direction at a constant speed for $375 \mathrm{~ms}$, abruptly changes both speed and direction, and continues along the new direction at the new speed for another $375 \mathrm{~ms}$. In the aligned and misaligned conditions, the particle is a rectangle with aspect ratio $2: 5$. In the aligned condition, the rectangle is oriented such that the principal axis is always aligned with the direction of motion (thus, orientation changes when motion direction changes). In the misaligned condition, the rectangle is oriented such that the principal axis is always aligned with the initial direction of motion (orientation never changes).

(1) An object moving at a constant velocity must have been set in motion at some point, but, if this happened before the object was viewed, an observer could simply conclude than an external energy source was involved. For this reason, we expected constant-velocity trajectories to get low animacy ratings, even though they, like all the trajectories in our study, are feasible animate motions. 
direction of the initial motion path (and hence misaligned with respect to the motion direction during the second half of the trial). We speculated that this last condition would be perceived as inanimate, owing to observers' expectation that an object's axis of elongation and its motion direction are normally aligned (Morikawa 1999). ${ }^{(2)}$

\section{Method}

\subsection{Subjects}

Participants were twenty-four college students recruited from two advanced undergraduate psychology classes. All had normal or corrected-to-normal vision.

\subsection{Apparatus and stimuli}

Stimuli were presented on a computer screen, oriented horizontally (flat on the floor) and bordered by a circular annulus. Subjects viewed the screen from above, in order to minimize the ascription of a gravitational frame (Bingham et al 1995). Viewing distance was approximately $95 \mathrm{~cm}$.

On each trial, a small white target entered the viewing area, moving across a uniform dark background in a random direction at a constant velocity of $2.84 \mathrm{~cm} \mathrm{~s}^{-1}$ $\left(1.71 \mathrm{deg} \mathrm{s}^{-1}\right)$. Targets were either dots with diameters of $0.20 \mathrm{~cm}$, subtending $0.123 \mathrm{deg}$ (dot condition), or rectangles $0.20 \mathrm{~cm} \times 0.08 \mathrm{~cm}, 0.123 \mathrm{deg}$ long by $0.054 \mathrm{deg}$ wide (aligned and misaligned conditions). Halfway through each trial, the target abruptly changed both speed and direction, continuing at the new speed and direction until the end of the trial. Direction changed $0^{\circ}, 10^{\circ}, 20^{\circ}, 40^{\circ}$, or $80^{\circ}$ towards either the left or the right (with respect to the initial motion direction) as determined randomly on each trial. Speed changed to $0.5,1,2$, or 4 times the initial speed.

\subsection{Procedure}

Subjects were instructed to pretend that they had just been hired as laboratory technicians by a government agency to help determine which of a set of microscopic particles were alive and which were not. The experimenter explained that the monitor displaying the stimuli should be thought of as a super-sized microscope, and that the displays represented the two-dimensional movement of tiny particles. Subjects were asked to rate each trial on a $1-7$ scale $(1=$ definitely not alive, $7=$ definitely alive), and were further instructed to give a low rating to any particle whose motion seemed "artificial, mechanical, or strange". Ten practice trials were provided, to familiarize the subjects with the stimuli. Following these practice trials, every subject viewed six blocks of trials, with each block containing all combinations of shape/alignment, direction change, and speed change $(3 \times 5 \times 4=60$ trials per block). Individual ratings from each trial (defined by shape/alignment, direction change, speed change, block, and subject) were entered into the statistical analysis with subject as the random variable.

\section{Results}

All three principal factors significantly influenced ratings of animacy (results are plotted in figure 2). Animacy ratings were highest in the aligned condition, intermediate in the dot condition, and lowest in the misaligned condition $\left(F_{2,46}=124.445 ; p<0.001\right)$.

${ }^{(2)}$ Of course, animate objects are not restricted to moving along their principal axes: in fact, humans move perpendicular to their elongation axes whenever they walk on two feet. Furthermore, many inanimate objects (eg javelins, footballs, trucks, etc) also maintain alignment between their heading and their principal axis. Hence, it is not simply a misalignment, but rather a failure to maintain an initial, presumably preferred, relationship between principal axis and heading which we expected to bias our observers against animate percepts. In other words, failing to re-orient when changing direction suggests a lack of control over body position, which hinders the percept that the motion is intentionally self-generated. 


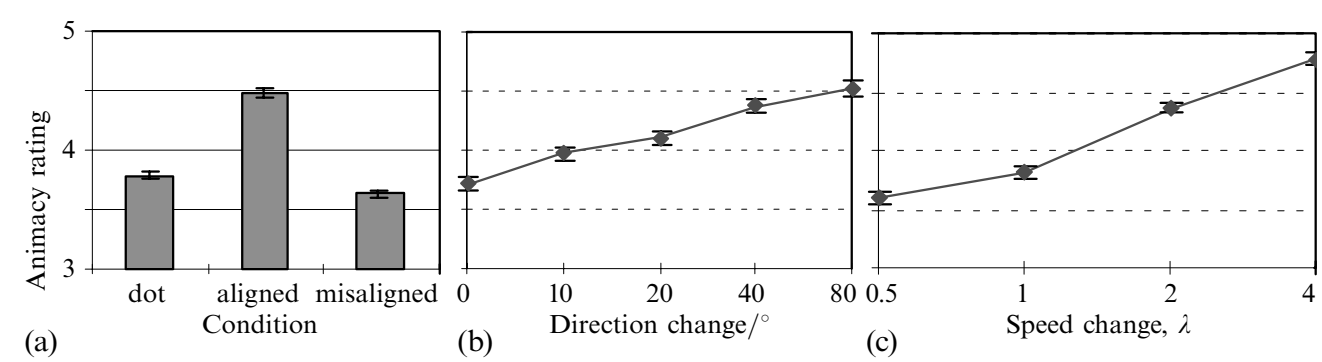

Figure 2. Effects of three main factors upon animacy ratings. (a) Shape/alignment condition. (b) Direction change. (c) Speed change. The aligned condition was rated significantly higher than the dot condition, and the misaligned condition significantly lower than the dot condition, as seen in (a). Animacy ratings increased with increasing changes in direction, as seen in (b), and with increases in changes in speed as seen in (c), where speed change is computed as the ratio, $\lambda$, of final speed, $V_{\mathrm{f}}$, to initial speed, $V_{0}$.

By design, the misaligned condition involved an anomalous relationship between principal axis orientation and motion direction during the second half of its trajectory, so this condition gave the weakest impression of animacy; hence, we excluded it from subsequent analyses. In the two remaining conditions, animacy ratings increased monotonically with increasing direction change $\left(F_{4,92}=9.024 ; p<0.001\right)$ and with increasing speed change $\left(F_{3,69}=4.935 ; p=0.004\right)$ (see figure 2$)$. Thus objects that accelerated the most rapidly and changed direction the most acutely were rated as the most animate. Generally, objects that decelerated were judged the least animate, even less so than those with no speed change.

All three pairwise interactions were significant (shape/alignment by speed change: $F_{3,69}=5.721, p=0.001$; shape/alignment by direction change: $F_{4,92}=12.668, p<0.001$; direction change by speed change: $F_{12,276}=3.014, p=0.001$ ) (see figure 3). The effect of increasing speed was greater in the dot condition than in the aligned condition, whereas the effect of increasing change in direction was greater in the aligned condition
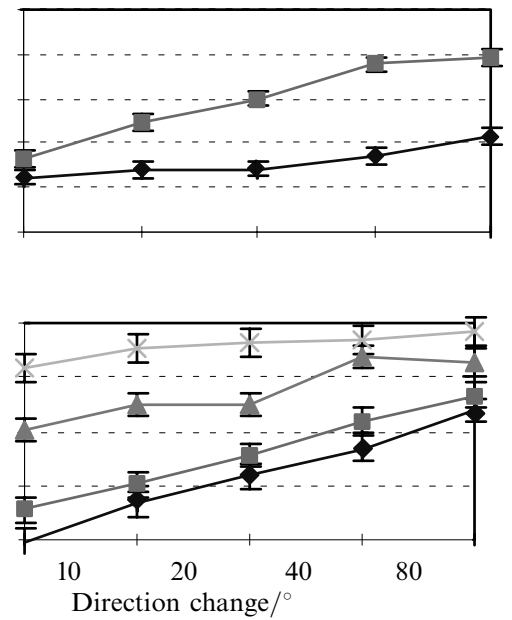

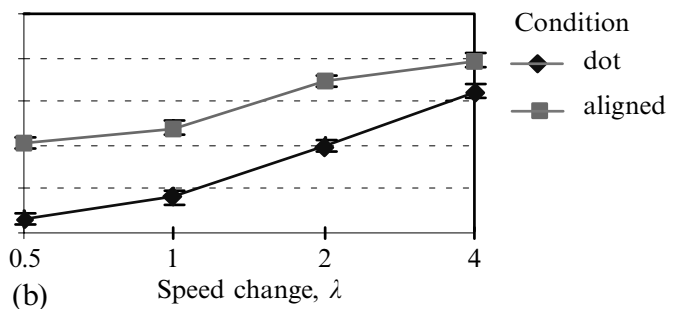

Speed change, $\lambda$

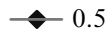

$=-1$

$-2$

$\times 4$

Figure 3. Two-way interactions among three main factors. (a) Shape/alignment condition by direction change. (b) Shape/alignment condition by speed change. (c) Speed change by direction change. Increases in direction change had more effect upon the aligned condition than the dot condition, as shown in (a). Increases in speed had more effect upon the dot condition than the aligned condition as shown in (b). Finally, (c) shows that increases in direction change had less of an effect for higher speed changes (particles with $\lambda>1$ ). 
than in the dot condition. This is consistent with the idea that in the aligned condition, changing direction really entails two distinct cues to animacy: the target's change in motion direction, and its change in bodily orientation. In the dot condition only the first of these is operative, leading to a smaller effect of direction change, and greater reliance on speed change. Finally, the speed change by direction change interaction showed that larger direction changes caused greater increases in animacy ratings for displays with lower final speeds than displays with higher final speeds.

The 3-way interaction between shape/alignment condition, speed change, and direction change was also significant $\left(F_{12,276}=2.076 ; p=0.019\right)$ (see figure 4$)$. In the aligned condition there were steady increases in ratings as the amount of direction change increased, even though the average rating increases were smaller in conditions where speed increased than when it remained constant or decreased. In contrast, in the cases where speed increased in the dot condition, increasing the amount of direction change did not produce monotonic increases of animacy ratings.
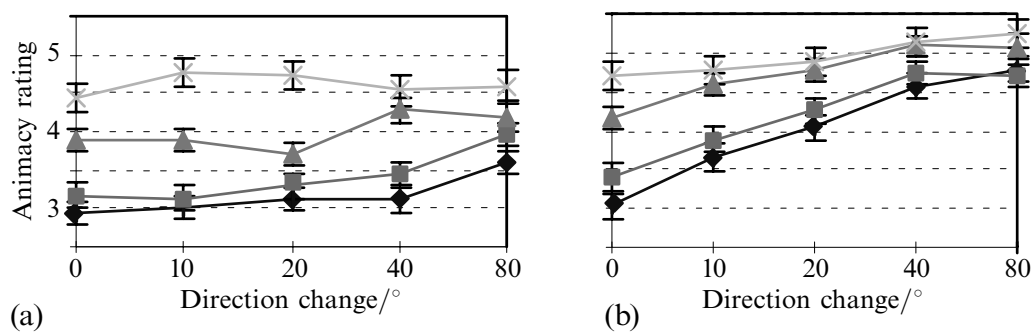

Speed change, $\lambda$

$\multimap 0.5$

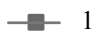

$-2$

$\times 4$

Figure 4. Three-way interaction between shape/alignment condition, speed change, and direction change. (a) Dot condition: interaction between speed change and direction change. (b) Aligned condition: interaction between speed change and direction change. The reduction in influence of direction change in cases where the speed increased (when $\lambda \geqslant 1$ ) was larger in the dot condition than the aligned condition. In fact, when speed increased in the dot condition, changes in direction had very little effect upon animacy ratings.

\section{Discussion}

Our study demonstrates that short, simple motion paths that contain a simultaneous change in speed and direction in the absence of any visible features that might explain this velocity change can convey an impression of animacy. The perception of animacy is strongest with large changes in direction, large increases in speed, and when the target is elongated in one direction and its elongation axis always remains aligned with its motion direction. In such displays, the target object gives an impression of volitional control over its motion path, a capacity normally exhibited only by living things. Our findings are consistent with, but extend, those of Dittrich and Lea (1994), who suggested that the perception of animacy is under the control of two factors: an impression of intentionality, and interaction between a target and its (possibly invisible) goal. Our study shows that the two factors interact: low-level aspects of a target's motion path can, in and of themselves, suggest an interaction with an (unseen) second entity (goal, predator, prey, etc), conveying an impression of intentionality, and thus of animacy. In short, our work suggests that perception of intentionality, and hence of animacy, can be relatively immediate and 'bottom - up'.

Our explanation of our data includes the claim that our subjects attribute objectives to the target particles. One may question why observers presented with our displays infer unseen prey or goals motivating a particle's movement instead of an unseen entity acting upon the particle. One explanation of why a goal might not be seen by an observer is that it is far away, outside of the observer's visible range. In contrast, an external agent which causes a particle to change velocity must contact the particle, hence would have to be 
inside the visible range when the velocity of the particle changed. Another reason that the goal of a particle might not be visible to an observer is that, since the particle is much smaller than the observer, the former may be able to detect things too small for the latter to see. For these (or for other) reasons it may be easier to explain why goals are not visible than why some object acting upon a particle is not visible.

In our experiment, the strongest impression of animacy occurred on trials in which the object accelerated, and hence in which observable motion energy was not conserved. However, our data demonstrate that animate percepts are not caused solely by failures of energy conservation (Stewart 1982), since acceleration yielded greater animacy ratings than deceleration. (3) Moreover, animate percepts cannot be entirely due to increases in energy as has been claimed (Bingham et al 1995) because animacy judgments were higher in the dot condition than in the misaligned condition, even though these two conditions entail precisely the same energy profiles. In both these conditions, the particle changed speed and direction without changing alignment. (In contrast, displays in the aligned condition, which produced even higher ratings, might have different energy profiles because an unknown amount of torque is required to rigidly rotate a particle.) In short, a variety of cues other than energy increases can contribute to the impression that a moving object is animate, at least including sensitivity to shape (demonstrated by the significant difference between the aligned condition and the dot condition) and sensitivity to apparent coordination between motion path and bodily orientation (demonstrated by the significant difference between the aligned condition and the misaligned condition).

Our work indicates that having a visible motion component that fails to conserve energy is not sufficient for a moving object to produce an impression of animacy. However, we propose that it is necessary; that is, that observers only perceive animacy when an object's motion appears not to conserve energy. Hence, we assume that subjects must be sensitive to violations of Newtonian laws (ie violations of energy conservation). Kaiser and Proffitt (1987) investigated this issue in their study of observers' ability to detect 'dynamic anomalies'. It is interesting to compare their findings to ours; one might expect the point at which their observers detect deviations from a natural, Newtonian motion path to be the point where our observers begin to attribute animacy to a moving particle.

In their study, Kaiser and Proffitt (1987) used stimuli modeled after a billiard-ball collision: one circle collided into another initially stationary circle, and both moved away after the collision. Kaiser and Proffitt manipulated several factors, including the direction that the initially-moving-ball followed after the collision. They reported that as long as the post-collision direction of this ball did not deviate from the expected, Newtonian direction by more than $25^{\circ},{ }^{(4)}$ subjects accepted their displays as a 'natural' motion event. In contrast, when the directional deviation of the ball from the expected path exceeded $25^{\circ}$, subjects labeled the event anomalous.

Kaiser and Proffitt determined the maximum 'tolerated' deviation using stimuli containing moving balls whose speeds never varied from the ones dictated by Newtonian laws. Hence, it is most appropriate to compare their findings to the subset of our dot-condition trials that did not involve a speed change. The relationship between animacy rating and direction change for these trials is plotted in figure 5. This plot reveals that dot-condition displays that contain direction changes below the maximum tolerated deviation reported by Kaiser and Proffitt (that is, displays with direction

(3) Although subjects can infer that gradual decelerations are caused by friction between a particle and an invisible surface, this type of motion still involves a hidden energy transfer. The crucial difference is that, in such cases, an inanimate explanation of the hidden transfer is apparently more plausible.

(4) The same manipulation was also tested in a condition where subjects were told that the system was frictionless. In this case, the maximum deviation tolerated by subjects was $20^{\circ}$. 


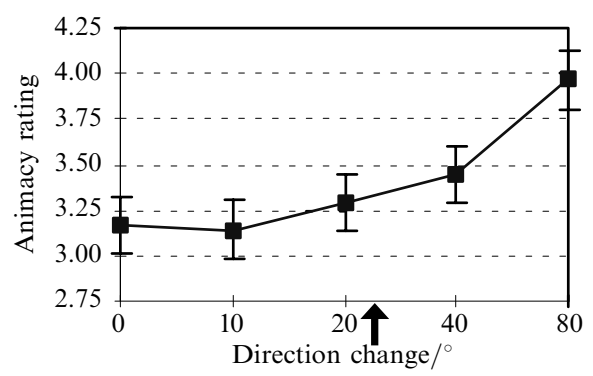

Figure 5. Effect of direction change in dot-condition trials with no speed change. This graph shows aggregate data for all twenty-four subjects. The arrow on the $x$ axis shows the maximum amount of unexpected direction change $\left(25^{\circ}\right)$ tolerated by Kaiser and Proffitt's (1987) subjects before they labeled a motion stimulus anomalous.

changes of $0^{\circ}$ or $10^{\circ}$ ) were rated lower than displays with direction changes above this maximum (displays with direction changes of $40^{\circ}$ or $80^{\circ}$ ). The displays with direction changes nearest Kaiser and Proffitt's threshold of $25^{\circ}$ were given ratings by our subjects very near the middle of the animacy-rating range (mean response $=3.29$ ), suggesting good agreement between their Newtonian-violation threshold and our animacy judgments.

Note that the graph in figure 5 and Kaiser and Proffitt's estimations of the maximum tolerated deviation are both based upon aggregate data, and hence conceal any individual differences in the amount of directional deviation which is accepted as natural in inanimate motion. Four plots, each showing a single subject's animacy ratings for dot-condition trials without speed changes are shown in figure 6 . These data indicate that there may be individual differences in using direction changes to infer animacy.

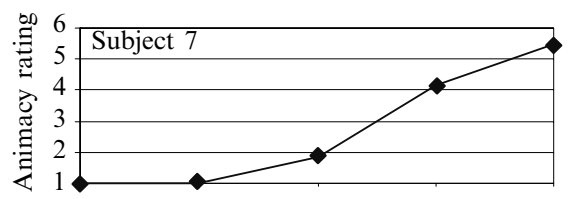

(a)

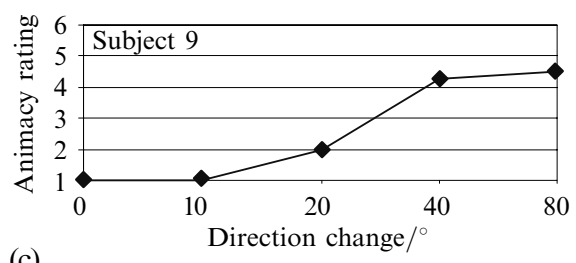

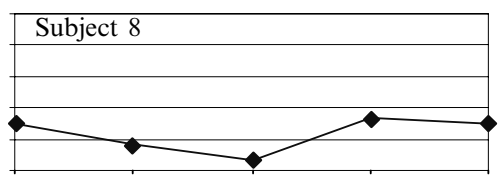

(b)

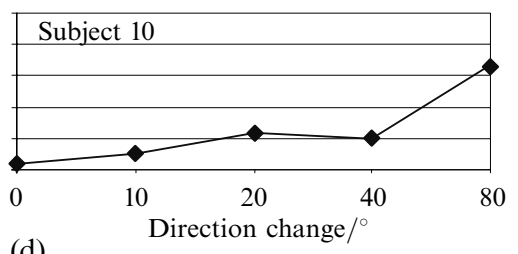

(d)

Figure 6. Animacy ratings showing the effect of direction change in the subset of dot condition trials with no speed change: individual data from four subjects. The ratings of subjects 7 and 9 are consistent with their tolerating inanimate objects having motion paths with unexpected direction changes of up to $20^{\circ}$, but for direction changes of $40^{\circ}$ or more rejecting the motion as inanimate and rating it animate. This is consistent with these subjects having similar sensitivity, and responses, to direction change. On the other hand, the data for subject 8 suggest that this subject does not have particular direction-change criteria for rejecting a display as an inanimate motion event; in effect, this subject rated all displays without speed changes as inanimate. Finally, the data for subject 10 (this subject rated displays low unless direction changed by $80^{\circ}$ ) are consistent with this subject having a higher tolerance for inanimate objects undergoing unexpected direction changes.

\section{Conclusion}

We regard animacy interpretation as a kind of "unconscious inference to the best explanation"; animacy is inferred when observable aspects of the display cannot easily be explained as ordinary inanimate motion. Hence animate cues might include any behavior that in some way suggests the presence of intentions, goals, perceptual competence, or any other capacity ordinarily exclusive to animals. Simultaneous speed and direction changes (not explained by contact with any other entities or environmental features) are a particularly simple and particularly perceptible example of such 
cues, and are 'minimal' in the sense that when they go to zero the resulting displayan object moving at constant velocity-produces little or no animate impression (as confirmed by our study). A default presumption of inanimacy is necessary to explain why animacy is not perceived ubiquitously in everyday scenes, eg constant-velocity motion signals produced by ego-motion, objects rolling down inclines or falling off surfaces, etc. However, the inanimate default is consistent with the simultaneous existence of other cues specific to inanimacy. Some of our subjects informally reported that the object in the misaligned condition appeared to have been 'struck' or 'kicked.' The apparent passive response to impact (ie failing to align its axis with the new motion direction) might be an example of a positive cue to inanimacy.

Although subjects do not seem to be consciously aware of the many subtle interactions among motion factors, it is not clear to what extent these effects are truly perceptual or involve unconscious cognitive decision processes. Under ordinary circumstances, the raw judgment of animacy studied here might be combined with more consciously accessible aspects of the context and semantics of a scene to produce a conscious decision about the nature of the observed object. This decision process would not be based upon motion alone (Gelman et al 1995). Again, it follows that the displays studied here are certainly not the only type that might lead to animate judgments; rather, they represent only a particularly simple kind of minimal case. Further study is required to investigate other varieties of displays that might yield similar percepts.

Acknowledgements. This work was supported in part by NSF SBR-9875175. We are grateful to Jim Davis, Rachel Gelman, Richard Mann, Shaun Nichols, Whitman Richards, Chuck Schmidt, and Maggie Shiffrar for many valuable discussions, and appreciate the helpful suggestions provided by two anonymous reviewers.

\section{References}

Bassili J N, 1976 "Temporal and spatial contingencies in the perception of social events" Journal of Personality and Social Psychology 33 680-685

Bingham G P, Schmidt R C, Rosenblum L D, 1995 "Dynamics and the orientation of kinematic forms in visual event recognition" Journal of Experimental Psychology: Human Perception and Performance 211473 - 1493

Chatterjee S H, Freyd J J, Shiffrar M, 1996 "Configural processing in the perception of apparent biological motion” Journal of Experimental Psychology: Human Perceptual Performance 22 $916-929$

Dasser V, Ulbaek I, Premack D, 1989 “The perception of intention” Science 243 365-367

Dittrich W H, 1993 "Action categories and the perception of biological motion" Perception 22 $15-22$

Dittrich W H, Lea S, 1994 "Visual perception of intentional motion" Perception 23 253-268

Gelman R, 1990 "First principles organize attention to and learning about relevant data: number and the animate - inanimate distinction as examples" Cognitive Science 14 79-106

Gelman R, Durgin F, Kaufman L, 1995 "Distinguishing between animates and inanimates: Not by motion alone", in Causal Cognition: A Multidisciplinary Debate Eds D Sperber, D Premack, A J Premack (Oxford: Clarendon Press) pp 150-184

Heider F, Simmel M, 1944 "An experimental study of apparent behavior" American Journal of Psychology $57243-259$

Hoffman D D, Flinchbaugh B E, 1982 "The interpretation of biological motion" Biological Cybernetics $\mathbf{4 2} 195-204$

Johannson G, 1973 "Visual perception of biological motion and a model for its analysis" Perception \& Psychophysics $14202-211$

Kaiser M K, Proffitt D R, 1987 "Observers' sensitivity to dynamic anomalies in collisions" Perception \& Psychophysics $\mathbf{4 2} 275-280$

Lettvin J Y, Maturana H R, McCulloch W S, Pitts W H, 1959 "What the frog's eye tells the frog's brain" Proceedings from the Institute of Radio Engineering 471940 - 1951

Mandler J M, 1992 "How to build a baby II: Conceptual primitives" Psychological Review 99 $587-604$

Mann R, Jepson A, Siskind J M, 1997 "The computational perception of scene dynamics" Computer Vision and Image Understanding $65113-128$ 
Mather G, West S, 1993 "Recognition of animal locomotion from dynamic point-light displays" Perception $22759-766$

Michotte A, 1963 The Perception of Causality (New York: Basic Books)

Morikawa K, 1999 "Symmetry and elongation of objects influence perceived direction of translational motion" Perception \& Psychophysics $61134-143$

Premack D, 1990 "The infant's theory of self-propelled objects" Cognition 36 1-16

Shiffrar M, Lichtey L, Chatterjee S H, 1997 "The perception of biological motion across apertures" Perception \& Psychophysics $5951-59$

Spelke E S, Phillips A, Woodward A L, 1995 "Infants' knowledge of object motion and human action", in Causal Cognition: A Multidisciplinary Debate Eds D Sperber, D Premack, A J Premack (Oxford: Clarendon Press) pp 44-78

Stewart J A, 1982 The Perception of Animacy PhD thesis, University of Pennsylvania

Webb J A, Aggarwal J K, 1982 "Structure from motion of rigid and jointed objects" Artificial Intelligence $19107-130$ 
